\title{
LE PROBLEME DE L'AUTRE
}

\section{DANS LA PHILOSOPHIE FRANCAISE}

\author{
DU VINGTIEME SIECLE
}

\section{Introduction}

Homo homini aut lupus, aut deus.

Plaute (Asinaria, II, 4, 88)

Tous les amateurs de la philosophie diraient sans doute avec Abbagnano qu' exister, c'est-à-dire être en tant qu'être humain, c'est philosopher. Seulement la philosophie n'est pas un lieu extraterrestre, dans lequel il faut chercher à fuir les difficultés, les ennuis et les échecs de la vie. Elle n'est pas un asile comme les jardins d'Epicure, ni non plus une activité aristocratique, réservée strictement pour une élite intellectuelle. Il faudrait chercher son véritable sens dans la conception de Platon selon laquelle Etre c'est être philosophe. "Philosopher," souligne Abbagnano, "cela veut dire d'abord que l'homme doit approcher son destin les yeux ouverts, c'est-à-dire élucider les questions, qui dérivent de la relation à soi, aux autres et au monde" $(1,11)$. Il s'ensuit par-là:

1) que le rapport à l'Autre est une relation existentielle fondamentale dont nous dépendons dans notre Etre et sans laquelle nous ne saurions être véritablement nous-mêmes et

2) que l'autre n'est pas un thème philosophique parmi d'autres mais qu'il constitue une partie centrale du champ philosophique basé sur l'existence. C'est sans doute la raison qui a amené Emmanuel Mounier à affirmer que "le problème de l'autre est une des grandes conquêtes de la philosophie existentielle" $(23,109)$ à laquelle il rattachait en ce tempslà sa propre "philosophie de la personne" $(23,12)$. Mais à vrai dire le problème de l'Autre est bien avant la philosophie existentielle une conquête de la philosophie française qui, du temps de Montaigne à nos jours, a toujours été concentrée sur "le phénomène humain" (T. de Chardin) et les problèmes de l'homme. La question fondamentale pour Montaigne, "Qui suis-je?", qui ne va pas cesser de préoccuper les philosophes français, va devenir au XXème siècle le point de départ de 
la pensée de Gabriel Marcel, en renvoyant par nécessité à l'Autre. Mais l'assertion de Mounier que la philosophie classique a laissé ce problème "dans un étrange délaissement" $(23,109)$ est fausse encore pour une autre raison. Le problème de l'autre occupait déjà une place importante dans la pensée de l'antiquité. Le mot même de religion qui renvoit à religare, qui veut dire unir, renferme cette idée de la relation du moi à l'autre. Ainsi l'idée de l'union devient-elle essentielle pour tous les systèmes philosophico-religieux et se trouve au fond de tous les livres sacrés qu'il s'agisse des Védas, des Brahmanas, de la Bhagavad Guita, de la Zend Avesta, du Livre des morts des anciens égyptiens ou celui des hindous, du Livre thibéthéen de la vie et de la mort, du Tao Te King des Paroles de Bouddha ou des Préceptes d'or de Pythagore. On la retrouve aussi dans les principes cosmiques de l'Amour et de la Discorde chez Empédocle, dans le thème de l'amitié chez les épicuriens et les stoiciens, ainsi que dans la conception d'Aristote que l'homme en tant que creation des parents est l'image de l'union de deux personnes. Mais sans doute le problème de l'autre est-il apparu au plus clair dans le christianisme en devenant la loi "nouvelle" et l'essence du Nouveau Testament. Ainsi à la question quel est le premier de tous les commandements, Jésus réponditil: "le premier de tous les commandements est: Ecoute Israel, le Seigneur, notre Dieu est le seul Seigneur; et tu aimeras le Seigneur ton Dieu de tout ton coeur, de toute ton âme, de toute ta pensée, et de toute ta force. C'est là le premier commandement. Et le second, qui est semblable au premier, est celui-ci: $\mathrm{Tu}$ aimeras ton prochain comme toimême. Il n'y a point de commandement plus grand que ceux-ci" (Marc, 12:29-31). C'est cette loi double de l'Amour qui est sans doute la clé de l'Autre tout à fait autre que nous-mêmes et pourtant intimior intimo meo, comme disait St-Augustin, ainsi que de l'autre en tant que semblable à moi par sa condition. C'est sans doute d'elle que se sont inspirés tous les courants philosophiques de tendance chrétienne.

\section{Les interpretations philosophico-religieuses de l'Autre}

Bien que subissant des influences de la part de penseurs comme Hegel, Marx, Dostoievsky, Nietzsche, Kierkegaard, Freud, Husserl, Scheler, Heidegger, Jaspers et Buber, les philosophes français ont essayé de trouver des réponses propres aux "problèmes éternels" (Dostoievsky), qui apparaissent précisément en face de l'Autre. C'est surtout dans ces conditions, que Jaspers nommait "Grenzsituationen" ("situations limites"), que l'Autre est devenu un thème fondamental dans la philosophie du XXème siècle, d'abord par un fait d'ordre historique, celui des deux 
guerres mondiales et de la guerre froide qui ont révélé l'Autre comme ennemi et comme l'autre face de l'humanité-l'inhumain--et ensuite par un fait historique, "la revolution socratique", ou anthropologisation constante de la philosophie contemporaine $(29,597)$. Devant ce défi pour la raison, les philosophes d'orientation religieuse ont essayé d'argumenter la thèse contraire à celle de "l'état de guerre" inévitable entre les hommes. Un tel effort fut sans doute la conception de Henri Bergson de l'amour mystique de l'humanité.

D'après Bergson, il y a dans l'homme deux especes d'émotion-l'une infra-intellectuelle, qui n'est qu'une agitation consécutive à une représentation, l'autre supra-intellectuelle et mystique $(9,270)$. L'émotion supra-intellectuelle qui a habité tous les mystiques est analogue à l'émotion créatrice du poète, mais plus élevée qu'elle, et a entrainé l'humanité au delà de la nature. C'est grâce à elle que l'homme a pu se décentrer de son soi pour retrouver son centre dans l'autre. Cette émotion essentiellement spirituelle n'est en fait que la charité comme participation de l'amour, essence de Dieu (9,34 et 270). Cet amour pour l'humanité étant très différent de l'amour familial et amical n'en est pas l'extension progressive. Comme formes de sociabilité primitive ces dernières nous portent à aimer un groupe fermé-notre groupe--tandis que pour aimer l'humanité entière il faut remonter par delà de cette nature primitive de l'instinct vers une liberation dont Dieu seul peut être l'auteur. L'amour mystique de l'humanité--souligne Bergson dans Les deux sources de la Morale et de la Religion--

\begin{abstract}
ne prolonge pas un instinct, il ne dérive pas d'une idée. Ce n'est ni du sensible ni du rationnel. C'est l'un et l'autre implicitement, et c'est beaucoup plus effectivement. (...) Coincidant avec l'amour de Dieu pour son oeuvre, amour qui a tout fait, il livrerait à qui saurait l'interroger le secret de la création. Il est d'essence métaphysique encore plus que de morale. Il voudrait, avec l'aide de Dieu, parachever la création de l'espece humaine et faire de l'humanité ce qu'elle eut été tout de suite si elle avait pu se constituer définitivement sans l'aide de l'homme lui-même. $(9,251)$
\end{abstract}

On retrouve cette même idée de l'évolution humaine par et à travers l'autre et l'amour de l'autre dans la Christologie de Pierre Teilhard de Chardin.

D'après Chardin la cosmogenèse passe par trois stades qui sont le stade prévital, le stade vital et le stade spirituel. Dans le premier évoluent les éléments chimiques, les galaxies et se forme l'enveloppe de 
la terre. Dans le second apparaissent l'enveloppe vivante de la terre--la biosphère--et tous les organismes, des plus simples aux plus complexes tels que l'homme. Le troisième stade implique le développement historique de l'homme qui se caractérise de nos jours par la formation de l'humanite--la noosphère ou sphère de l'Esprit--qui rendrait possible le passage au point Oméga: le centre suprapersonnel de l'Univers. Si l'époque contemporaine représente l'étape finale de la socialisation, Chardin n'ignore pourtant pas les grandes difficultés auxquelles se heurte l'humanité dans cet effort d'unité. D'après lui toutes les aspirations d'union jusqu'à présent ont échoué parce qu'elles avaient choisi une mauvaise base. Les unions faites sur le fond d'intérêts matériels ou de divertissement se sont vite avérés comme instables et momentanés. La raison profonde c'est que la condition ultime de l'union humaine réside dans le Corpus Christi, ce Milieu Divin qui est en nous et hors de nous et qui attend un complément essentiel de la réunion de chacun avec les autres. Dans une belle page Teilhard de Chardin l'illustre ainsi:

\begin{abstract}
Un, définitivement, dans le Plérome, le Milieu Divin doit commencer à devenir un dès la phase terrestre de notre existence. Lors même donc que le Chrétien avide de vivre en Dieu aurait donné à ses désirs toute la pureté, à ses prières toute la foi, à son action toute la fidélité possible, d'immenses possibilités s'ouvriraient encore à la divinisation de son Univers. Il lui resterait à raccorder son oeuvre élémentaire à celle de tous les ouvriers qui l'entourent (...). Il lui faut réchauffer sa propre chaleur à celle de tous ces foyers (...), se mettre à la température et la tension communes. (...). A quelle force est-il donné de fondre et d'exalter nos rayonnements partiels dans le rayonnement principal du Christ? A la Charite, principe et effet de toute liaison spirituelle. La charité chrétienne, si solennellement prêchée par l'Evangile, n'est pas autre chose que la cohésion plus ou moins consciente des âmes, engendrée par leur convergence commune in Christo Jesu. Impossible d'aimer le Christ sans aimer les autres (dans la mesure où ces autres vont vers le Christ); et impossible d'aimer les autres (dans un esprit de large communion humaine) sans se rapprocher du Christ par le même mouvement. (...). Cette conjonction inévitable s'est toujours traduite, dans la vie intérieure des Saints, par un débordement d'amour pour tout ce qui, dans les créatures, porte en soi un germe de vie éternelle (37, 182-183).
\end{abstract}

Mais cet amour, cette Charité sont-ils si faciles? Il est curieux que pareil à un des héros de Dostoievsky qui constate qu'il lui est possible d'aimer l'humanité comme idée abstraite, mais non les hommes concrets, Teilhard de Chardin lui-même constate en toute sincérité: 
Mon Dieu, je vous l'avoue, j'ai bien longtemps été, et je suis encore hélas, réfractaire à l'a mour du prochain. Autant j'ai ardemment goaté la joie surhumaine de me rompre et de me perdre dans les âmes auxquelles me destinait l'affinité bien mystérieuse de la dilection humaine--autant je me sens nativement hostile et ferme en face du commun de ceux que vous me dites d'aimer. Ce qui, dans l'Univers, est au-dessus ou dessous de moi (...), je l'intègre facilement dans ma vie intérieure: la matière, les plantes, les animaux, et puis les Puissances, les Dominations, les Anges, je les accepte sans peine, et je jouis de me sentir soutenu dans leur hierarchie. Mais 'l'autre,' mon Dieu,--non seulement 'le pauvre, le boiteux, le tordu, l'hébeté,' mais l'autre simplement, l'autre tout court,--celui, qui par son Univers en apparence fermé au mien semble vivre indépendamment de moi, et briser pour moi l'unite du silence du Monde,--serais-je sincere si je disais que ma reaction instinctive n'est pas de le repousser? et que la simple idé d'entrer en communication spirituelle avec lui ne m'est pas un dégout? (37, 184-185).

La réponse à cette question, assez rhétorique d'ailleurs, est à trouver dans la prière adressée par Chardin tout de suite après:

Mon Dieu, dit-il, faites pour moi, dans la vie de l'Autre briller votre Visage (...). Donnez moi de vous apercevoir, même et surtout, au plus intime, au plus parfait, au plus lointain de l'âme de mes frères" (37, 185).

Car, Chardin se rend bien compte que ce que le Verbe veut est bien supérieur à une simple sympathie personelle; c'est l'attrait vers "l'Autre" des affinités combinées d'un monde pour lui-même et de ce monde pour Dieu. Et c'est en cela que consiste le salut de l'unité humaine dans le Christ comme "océan inconnu de la charité."

Sur cet amour précisément inconnu parce qu'infini va s'accentuer La philosophie morale de Jacques Maritain qui trouve sa justification aussi bien dans l'Evangile que dans la maxime de Dostoievsky, "Crois que Dieu t'aime d'une façon que tu ne peux pas imaginer."

Pour Maritain tout grand système moral est en réalité un effort, pour demander à l'homme de dépasser, de transcender en quelque façon sa condition naturelle. Cet effort aboutit, d'après lui, seulement avec le christianisme; d'abord, comme comportement qui est réalisé à peu près complètement chez les saints, et, ensuite, comme don de la grâce accordée à ceux qui consentent activement et par amour. Mais si la grâce fait participer l'homme à la vie divine et si elle surélève sa nature 
dans l'ordre propre de celle-ci, cependant c'est une nature toujours blessée qui est ainsi surélevée, c'est un homme toujours dévoré de faiblesse qui a part à la vie éternelle et à l'amitié de Dieu. La question de la souffrance se pose avec d'autant plus de gravité que, d'une part, en tant que signature de leur amour et de coopération à l'oeuvre du Christ les souffrances sont voulues par les saints comme le bien le plus précieux d'ici-bas et, d'autre part, le reste des hommes a cherché à les diminuer, à apaiser le mal dont nous souffrons avec les autres. La réponse, c'est que de soi la souffrance est vraiment un mal, et restera toujours un mal. Si elle peut être transvaluee---souligne Maritain--ce n'est que "dans le feu de l'amour actuel, et absolument incommunicable, entre le soi d'un homme et le Soi divin; et cela reste un secret fermé, valable seulement pour la subjectivité individuelle" $(21,571)$. Mais alors, comment pourrions-nous prendre notre parti dans la souffrance des autres? Jésus n'avait-il pas pleuré sur la mort de Lazare et sur la douleur de Marthe et de Marie? C'est que la lutte contre la souffrance est un des aspects de l'effort par lequel l'humanité avance. Et pourtant, à mesure qu'on gagne le terrain dans le combat contre la souffrance, de nouvelles causes de souffrance se mettent à foisonner, de telle sorte que l'homme, en dépit de tous ses progrès, n'en aura jamais fini avec la souffance comme il n'en aura jamais fini avec la maladie. Bien que sur ce point Maritain se réfere à l'oeuvre de René Dubos, Mirage of Health (New York: Harper, 1959), il rappelle au fait l'argument le plus fort des existentialistes athées: "L'homme--constate Camus--ne peut maîtriser en lui tout ce qui doit l'être. Après quoi, les enfants mourront toujours injustement, même dans la société parfaite. Dans son plus grand effort, l'homme ne peut que se proposer de diminuer arithmétiquement la douleur du monde. Mais l'injustice et la souffrance demeureront et, si limitées soient-elles, elles ne cesseront pas d'être le scandale. Le "pourquoi?" de Dmitri Karamazov continuera de retentir; l'art et la révolte ne mourront qu'avec le dernier hommen $(21,706)$. Comment répondre à ce scandale de mal, de la souffrance et de la mort? Et Maritain n'avoue-t-il pas qu' "il restera toujours assez de mal et de peine pour mettre dans l'angoisse le coeur et l'intelligence" (21, 572)? Mais tout cela, c'est pour souligner que les réponses des philosophes à cette question ne sont pas suffisantes et qu'il existe une autre réponse qui dépasse la vie terrestre--celle qui a été donnée dans le Sermon sur la montagne. "S'il y a dans l'humanité une masse immense de douleur--ecrit Maritain--(...) c'est pour qu'elle soit rachetée, et que partout du moins où la liberté humaine n'oppose pas son refus, ceux qui ont pleuré dans nos vallées soient consolés pour toujours" (ibid). 
A la différence de cette réponse purement évangélique, la pensée personnaliste, l'existentialisme chrétien de Gabriel Marcel et le néojudaisme de Lévinas proposeront une argumentation philosophique de la possibilité d'intersubjectivité, de communication authentique et d'union.

En partant de l'existence comme ouverture tous les personnalistes français admettent que l'homme a une nature dialogique. "Le premier mouvement qui révèle un être humain dans la petite enfance-remarque Mounier--est un mouvement vers autrui: l'enfant de six à douze mois, sortant de la vie végétative, se découvre en autrui, s'apprend dans des attitudes commandées par le regard d'autrui. Ce n'est que plus tard, vers la troisième année, que viendra la première vague d'égocentrisme réfléchi" (25, III, 453). Et pourtant si on observe l'histoire on verra que la vie en société est une guérilla permanente. Les cheminements de la camaraderie, de l'amitie ou de l'amour semblent perdus dans cet immense échec de la fraternité humaine. "Le monde des autres n'est pas un jardin de délices. Il est une provocation permanente à la lutte, à l'adaptation et au dépassement. Il réintroduit constamment le risque et la souffrance là où nous touchions à la paix," constate Mounier (25, III, 451). Il y a donc un séparatisme et un individualisme profond qui traverse la civilisation moderne et en est la marque. C'est contre lui, qui a centré l'individu sur soi, que le personnalisme va établir son projet vers autrui.

Le fait primitif dont il faudrait partir, d'après Mounier, c'est que la personne, par le mouvement qui la fait être, s'ex-pose. Ainsi est-elle par nature communicable et elle est même la seule à l'être. Mais "lorsque la communication se relâche ou se corrompt, je me perds profondément moi-même--(...) alter devient alienus, je deviens, à mon tour, étranger à moi-même, aliéné. On pourrait presque dire que je n'existe que dans la mesure où j'existe pour autrui, et, à la limite: être, c'est aimer. Ces vérités--souligne Mounier--sont le personnalisme même, au point qu'il y a pléonasme à désigner la civilisation qu'il poursuit comme personnaliste et communautaire. Elles expriment, face à l'individualisme et à l'idéalisme persistants, que le sujet ne se nourrit pas par autodigestion, qu'on ne possède que ce qu'on donne ou ce à quoi on se donne, qu'on ne fait pas son salut seul, ni socialement, ni spirituellement" (25, III, 453). Autrement dit, la personne n'existe que vers autrui, elle ne se connaît que par lui, elle ne se trouve qu'en lui. C'est pourquoi "le tu, et en lui le nous, précede le je, ou au moins l'accompagne" (ibidem) et "l'acte d'amour est la plus profonde certitude 
de l'homme, le cogito existentiel irrefutable: J'aime, donc l'être est, et la vie vaut (la peine d'être vécue)" (25, III, 455). En même temps Mounier se rend bien compte que la communication ainsi conçue n'est qu'un idéal, qu'une idée au sens kantien. "L'être n'est pas amour du matin au soir" et c'est pour cela même que la communication subit plusieurs échecs tel que le fait que quelque chose d'autrui nous échappe toujours, que quelque chose en nous résiste foncièrement à l'effort de réciprocité, que quand on a formé une alliance de réciprocité elle nourrit bientôt un nouvel égocentrisme, etc. Mais bien que nous vivons dans un "monde cassé" (G.Marcel), Mounier n'accepte pas que la solitude est la condition humaine en tant que telle, car on n'est pas seul, on se fait seul. Cette conviction constituera un des fragments les plus caractéristiques de son "optimisme tragique."

On retrouve une conception analogue chez Maurice Nédoncelle pour qui autre ne veut pas dire non-moi, mais volonté de promotion mutuelle des moi, et par là même, transparence de l'un pour l'autre. La condition personelle est bipolaire de fait que le toi et le moi sont l'un par l'autre à la fois cause et effet; ils sont, d'un même coup, hétérogènes par leur posture et identiques par leur communaute. Cela est valable surtout pour la relation à Dieu, cet Autre qui forme avec le moi une conscience collegiale d'une forme nouvelle et unique. Dieu est ce moi idéal de toute conscience qui conduit la personne à la conclusion que la solitude du moi est toujours une illusion. Le nous que la conscience forme avec Dieu et par lequel elle tend à humaniser le Dieu qui la divinise, est à sa manière une oeuvre, une nouvelle création mutuelle. Mais plus pro-fondément encore, ce nous est lui-même le principe incrée, qui est en toute texture de la création déjà-là $(26,321)$. Par là même la réciprocité entre le moi et le toi s'avère double: réciprocité des consciences humai-nes et réciprocité humano-divine $(27,242-252)$. C'est la dernière qui est la condition ultime de l'existence, car "pour être soi, il faut être au moins deux et pour être pleinement soi, il faut que l'autre soit Dieu" $(28,101)$.

Cette tendance de traiter l'Autre comme l'intermédiaire à travers lequel on prend conscience de soi est très visible surtout dans la conception existentielle de Gabriel Marcel.

D'après Marcel la question "Qui suis-je" renvoit nécessairement à l'autre car "l'autre est déjà-là quand j'ai conscience pour moi-même" $(19, \mathrm{I}, 187)$. D'autre part si l'autonomie du moi est dans l'immanence, l'existence véritable est transcendance vers le monde et vers les autres. 
Par la "non seulement nous avons le droit d'affirmer que les autres existent, mais--dit-il--je serais disposé à soutenir que l'existence ne peut être attribuée qu'aux autres, en tant qu'autres, et que je ne puis me penser moi-même comme existant qu'en tant que je me conçois comme n'étant pas les autres" (19, I, 129-130). C'est pour cela que le moi "n'existe qu'en tant qu'il se traite lui-même comme étant pour autrui" (19, I, 130). En même temps Marcel s'oppose à la morale égocentrique qui traite l'autre en tant que le moyen de la realisation de soi-même ou, au contraire, comme son entrave. La personne s'affirme en tant que personne seulement dans la mesure où elle assume la responsabilité de ses actes et de ses paroles devant autrui. "Je tends à m'affirmer comme personne dans la mesure où, assumant la responsabilité de mes actes, je me comporte comme un être réel, participant à une certaine société réelle (...), je m'affirme comme personne dans la mesure où je crois réellement à l'existence des autres et où cette croyance tend à informer ma conduite" $(20,27)$. L'homme doit prendre conscience de cette unité "personne--engagement--communauté--réalité" pour la saisir comme un appel de l'être. Et dans la réponse à cet appel Homo Viator, l'homme en route vers l'au-delà, ne peut accéder à la véritable communauté qu'à travers les autres.

Pareil à Marcel le néojudaisme de Lévinas vise-t-il le mode de l'au-delà-de-l'être, resp. la relation à l'Autre comme Tout Autre, comme Transcendant et Infini (ou Dieu). Ce mode est d'après lui celui du temps. Comme modalité de l'être fini, le temps signifie la dispersion de l'être de l'étant en moments instables et fournit l'idée fulgurante de la vie et de la mort. Pourtant la thèse principale dans Le temps et l'Autre consiste non à penser le temps comme dégradation de l'éternité, mais comme relation à ce qui en tant qu'absolument autre ne se laisserait pas assimiler et comme infini ne se laisserait pas comprendre. Ainsi le "mouvement" du temps comme transcendance à l'infini du "tout Autre" ne se temporalise pas d'une façon linéaire. Sa façon de signifier, marquée par le mystère de la mort, fait un détour en entrant dans l'aventure éthique de la relation à l'autre homme. Ainsi le but principal énoncé dans Le temps et l'Autre "consiste à montrer que le temps n'est pas le fait d'un sujet isolé et seul, mais qu'il est la relation même du sujet avec autrui" $(15,17)$. Mais il se pose ici le probleme est-ce que la mort, annoncée comme autre, comme aliéna-tion de notre existence, ne va pas écraser la subjectivité, resp. est-ce que l'homme ne se laisserait pas écraser par l'autre? D'après Lévinas 
Le problème ne consiste pas à arracher une éternité à la mort, mais à permettre de l'accueillir, de conserver au moi, au milieu d'une existence ou un événement lui arrive, la liberté acquise par l'hypostase. Situation que l'on peut appeler la tentative de vaincre la mort, ot, à la fois, l'événement arrive et où cependant le sujet sans l'accueillir, comme on accueille une chose, un objet, fait face $a$ l'evénement $(15,66-67)$.

C'est précisèment cette situation où l'événement arrive à un sujet qui ne l'assume pas, qui ne peut rien pouvoir à son égard, mais où cependant il est en face de lui, qui est la relation avec autrui. Cette relation, c'est le face-à-face avec autrui, la rencontre d'un visage qui, à la fois, donne et dérobe autrui. "L'autre 'assumé--c'est autrui;" telle est la définition donnée par Lévinas $(15,67)$ qui se prolonge en une description, qui montre l'ambiguité de la notion d'autrui. Car si l'autre est connu d'abord par la sympathie comme un autre moi-même, comme alter ego, il n'est pas seulement un alter ego, il est ce que moi je ne suis pas, c'est-à-dire l'altérité même. "Il est, par exemple, le faible, le pauvre, 'la veuve et l'orphelin,' alors que moi je suis le riche ou le puissant. On peut dire que l'espace intersubjectif n'est pas symétrique," souligne Lévinas $(15,75)$. Cela explique pourquoi la vie sociale n'est jamais une harmonie parfaite, pourquoi il y a une telle complexité et tant de désordre dans la civilisation actuelle. Pourtant à la différence d'un Buber, Sartre ou Heidegger le but principal de Lévinas est de montrer que "l'altérité n'est pas purement et simplement l'existence d'une autre liberté à côté de la miennen $^{n}(15,87)$. Pour lui la coexistence de plusieurs libertés est une multiplicité qui laisse intacte l'unité de chacune. Plus encore, cette multiplicité s'unit en une volonté générale, à une transcendance temporelle d'un présent vers le mystère de l'avenir $(15,89)$.

\section{Les conceptions des athees}

A la différence des conceptions de tendance chrétienne qui révèlent un certain optimisme final dû à la croyance en un au-delà de l'histoire terrestre, resp. à l'avènement futur du royaume de la Justice, les existentialistes athees et certains penseurs postmodernes d'aujourd'hui essaient de fournir une esquisse et des analyses qui ne tiennent compte que de "ce qui est" sans "rien faire inventer qui ne soit certain" $(12,76)$.

Or, partir de ce qui existe de facto, cela conduit à des images moins belles et moins agréables de l'Autre qu'on ne le voudrait. Et telles sont sans doute les images refléchies dans les oeuvres de Sartre, de Camus et de Simone de Beauvoir. 
On peut s'imaginer le choc qui a causé dans les milieux religieux et bourgeois la conception sartrienne du Pour-Autrui qui constitue environ la moitié (300 pages) de L'être et le néant. En partant du cogito comme projet, Sartre souligne que l'homme qui est pour-soi est en même temps un pour-autrui. Autrement dit, "le Cogito de l'existence d'autrui se confond avec mon propre Cogito" $(33,297)$. Mais cela ne se fait pas à l'immédiat. L'homme découvre autrui au moyen du regard, en le concevant à la fois comme objet et comme être humain. Du point de vue visuel l'autre se présente à moi comme un objet parmi les autres, mais en même temps il differe d'eux, car il a aussi la possibilité de me voir. Le problème, c'est précisément que ce n'est pas seulement moi qui vois l'autre, mais aussi que l'autre me voit en me transformant par-là en objet. Je perds, de cette façon, la subjectivité et je saisis le regard de l'autre comme "aliénation de mes propres possibilités", comme une limitation de ma liberté, et ainsi "ma chute originelle, c'est l'existence de l'autre" (33, 309). La pensée de l'autre se transforme ici dans une maladie de laquelle l'homme ne pourrait, apparemment, se délivrer qu'en essayant de soumettre la liberté de l'autre. Les différentes voies de cette soumission sont d'apres Sartre l'amour, le langage, le masochisme, l'indifference, le désir, la haine et le sadisme. Les trois premières sont des attitudes primaires, tandis que les autres sont secondaires. L'amour n'est pas tellement un désir de posséder le corps de l'autre que la volonté de posséder sa liberté: "Ainsi l'amant ne désire-t-il pas posséder l'aimé comme on possède une chose; il réclame un type spécial d'appropriation. Il veut posséder une liberté comme libertén $(33,416)$. Mais ce projet finit toujours par échouer, car même si j'arrive à conquérir l'autre, du moment où il se met à m'aimer il me transforme en sujet en devenant lui-même objet et en m'échappant par-là. Par contre, quand je deviens indifférent je subis aussi l'échec car "non seulement je ne puis obtenir de lui ce que je veux, mais en outre cette quête provoque un évanouissement du savoir même qui concerne ce que je veux" $(33,432)$. II s'ensuit par-là que "le conflit est le sens originel de l'être-pour-autrui" $(32,413)$. Cette position extrémiste est affirmée par Sartre déjà dans Huis-clos avec la phrase devenue célebre: "L'enfer, c'est les autres" $(35,167)$. On sait qu'après avoir vu le spectacle Gabriel Marcel s'est écrié: "Le paradis, c'est les autres". Et Merleau-Ponty, pour sa part, affirma que la perception d'autrui ne faisait de problèmes qu'aux adultes, car l'enfant vit dans un monde qu'il croit d'emblée accessible à ceux qui l'entourent.

Autrui ou moi, il faut choisir, dit-on. Mais on choisit l'un contre l'autre, et ainsi on affirme les deux. Autrui me transforme en objet et 
me nie, je transforme autrui en objet et le nie, dit-on. En réalité le regard d'autrui ne me transforme en objet, que si l'un et l'autre nous nous retirons dans le fond de notre nature pensante, si nous nous faisons l'un et l'autre regard inhumain $(22,414)$.

De ce fait la liberté qui veut se conserver rejette la communication, mais ne l'annihile pas car "le refus de communiquer est encore un mode de communication" (ibidem.). Sans doute les critiques et les malentendus sur la conception de Sartre étaient si nombreuses, qu'il se sentit obligé d' atténuer ses positions en déclarant dans L'existentialisme est un humanisme que "je ne puis prendre ma liberté pour but, que si je prends également celle des autres pour but" $(33,83)$, ainsi que d'expliquer 20 ans plus tard: "'L'enfer, c'est les autres' à toujours été mal compris. On a cru que je voulais dire que nos rapports avec les autres étaient toujours empoisonnés, que c'étaient toujours des rapports interdits. Or, c'est tout à fait autre chose que je veux dire. Je veux dire que si les rapports avec autrui sont tordus, viciés, alors l'autre ne peut être que l'enfer (...). Les autres sont, au fond, ce qu'il y a de plus important en nous-même, pour notre propre connaissance de nous-même" $(13,101)$. Par cette intervention on voit aussi que ce "pessimisme" assigné à Sartre n'est pas définitif: "Quel que soit le cercle d'enfer dans lequel nous vivons--dit-il,-- je pense que nous sommes libres de le briser. Et si les gens ne le brisent pas, c'est encore librement qu'ils y restent. De sorte qu'ils se mettent librement en enfer" (ibidem.).

Cette même vision, ou encore plus pessimiste, de l'Autre comme une altérité opposée et hostile à moi est à retrouver chez Camus surtout dans L'étranger et Le mythe de Sisyphe. C'est face au monde et aux autres que l'homme découvre cette altérité absurde qui le renvoit à lui-même précisément comme un tout autre, comme un étranger. Camus écrit:

Dans un univers soudain privé d'illusions et de lumières l'homme se sent un étranger. Cet exil est sans recours, puisqu'il est privé des souvenirs d'une patrie perdue ou de l'espoir d'une terre promise (...) L'hostilité primitive du monde, à travers les millénaires, remonte vers nous (...) cette épaisseur et cette étrangeté du monde, c'est l'absurde. Les hommes aussi sécrètent de l'inhumain. Dans certaines heures de lucidité, l'aspect mécanique de leurs gestes, leur pantomime, privée de sens, rend stupide tout ce qui les entoure (..) Ce malaise devant l'inhumanité de l'homme même, cette incalculable chute devant l'image de ce que nous sommes, cette "nausée" comme l'appelle un auteur de nos jours, c'est aussi l'absurde. De même l'étranger qui, à certaines secondes, vient à notre rencontre dans une glace, le frère 
familier et pourtant inquiétant que nous retrouvons dans nos propres photographies, c'est encore l'absurde" (12, 28-29).

Ce n'est pas par hasard qu'une des meilleures explications de L'etranger est fournie par Sartre: "L'étranger qu'il (Camus - Y. R.) veut peindre dit Sartre, - c'est justement un de ces terribles innocents qui font le scandale d'une société parce qu'ils n'acceptent pas les règles de son jeu. II vit parmi les étrangers, mais pour eux aussi il est étranger. C'est pour cela que certains l'aimeront, comme Marie, sa maîtresse, qui tient à lui "parce qu' il est bizarre"; et d'autres le détesteront pour cela, comme cette foule des assises dont il sent tout à coup la haine monter vers lui. Et nous-mêmes qui, en ouvrant le livre, ne sommes pas familiarisés encore avec le sentiment de l'absurde, en vain chercherions-nous à le juger selon nos normes accoutumées: pour nous aussi il est un étranger" $(36,104)$.

Tout en suivant cette ligne, qui tend à peindre autrui comme un être hostile à nous, Simone de Beauvoir va joindre à cela deux dimensions nouvelles--celle des autres comme nécessaires et contribuantà notre réalisation, et celle de l'Autre entendu comme l'autre-de-l'homme, resp. la femme. La première tendance est exprimée dans son premier livre, L'invitee, dans lequel elle donne le recit d'une jeune femme, Françoise, qui a accueilli auprès d'elle et de son ami Pierre une "invitée", Xavière, qui se révèle insupportable. Xavière lui enlève Pierre, opprime ses rapports avec les autres et tourmente sa conscience. Alors Françoise décide de se débarrasser de celle qui la torture pour faire son choix--le choix de sortir de l'ombre de l'Autre pour être elle-même:

"Françoise était seule dans un ciel glacé. C'était une solitude si solennelle et si définitive qu'elle ressemblait à la mort. C'est elle ou moi. Ce sera moi. II y eut un bruit de pas dans la salle de bains. Xavière rentra dans sa chambre. Françoise marcha vers la cuisine et ferma le compteur à gaz. (...) Elle resta debout surveillant la porte de Xavière. Seule. Sans appui. Ne reposant plus que sur elle-même. Elle attendit un long moment, puis elle' entra dans la cuisine et posa la main sur le levier du compteur. Sa main se crispa. Ça semblait impossible. En face de sa solitude, hors de l'espace, hors du temps, il y avait cette présence ennemie qui depuis si longtemps l'écrasait de son ombre aveugle; (...). Et cependant il suffisait d'abaisser ce levier pour l'anéantir. Anéantir une conscience. Comment puis-je? pensa Françoise. Mais comment se pouvait-il qu'une conscience existât qui ne fut pas la sienne? Alors, c'était elle qui n'existait pas. Elle répeta: "Elle ou moi". Elle abaissa le levier. Elle rentra dans sa chambre (...) La porte de Xavière était fermée de l'intérieur. On croirait à un 
accident ou à un suicide. (...) Demain matin, elle sera morte. (...) Seule. Elle avait agi seule. Aussi seule que dans la mort. (...) Personne ne pouvait la condamner ni l'absoudre. Son acte n'appartenait qu'a elle. "C'est moi qui le veux." C'était sa volonté qui était en train de s'accomplir, plus rien ne la séparait d'elle-même. Elle avait enfin choisi. Elle s'était choisie" (3, 504).

Ce n'est que dans son essai Pour une morale de l'ambiguité qu'elle va affirmer que la morale existentialiste, c'est d'une part l'acceptation de la séparation des gens et, d'autre part, celle de leur liaison $(5,26)$. L'homme qui veut donner un sens à sa vie doit vouloir la liberté car l'existence est une passion. "On a trop souvent décrit son échec et l'enfer qu'il s'est fait lui-même--dit Beauvoir,--mais la passion se transforme en une liberté authentique seulement quand l'homme se consacre aux autres, sans vouloir les soumettre et les objectiver pour sauver sa liberté. Notre liberté exige, pour s'épanouir, son accomplissement dans un 'avenir ouvert' ou, autrement dit, ce sont les autres qui nous ouvrent notre avenir propre" $(5,116)$. Cette idée qui vise sans doute à modérer la conception sartrienne de l'autre se trouve aussi d'une façon implicite dans ses romans Tous les hommes sont mortels, Les mandarins e.a. (cf. 6 et 7). Mais l'apport principal et tout à fait originel à ce probleme se trouve sans doute dans Le deuxième sexe - l'essai qui a poussé tant de femmes à prendre conscience de leur condition et d'entrevoir des horizons d'émancipation.

D'après Beauvoir depuis l'époque biblique, la femme, qui fut conçue comme étant crée avec une côte d'Adam, a été traité comme un être non-autonome, dépendant de l'homme. Ainsi c'est l'homme qui fut toujours le Sujet, l'Absolu, par rapport auquel elle a été définie comme l'Autre $(8, \mathrm{I}, 15)$. "Même dans son image la plus sublime, celle de Vérité, Beauté, Poésie, elle est Tout: une fois de plus tout sous la figure de l'autre, Tout excepté elle-même" $(8, I, 375)$. Un exemple en est l'oeuvre d'André Breton. "La perspective de Breton étant exclusivement poétique--souligne Beauvoir-c'est exclusivement comme Poésie donc comme autre que la femme y est envisagé. Dans la mesure où on s'interrogeait sur son destin à elle, la réponse serait impliquée dans l'idéal de l'amour réciproque: elle n'a d'autre vocation que l'amour; ceci ne constitue aucune infériorité puisque la vocation de l'homme est aussi l'amour. Cependant on aimerait savoir si pour elle aussi l'amour est cle du monde, révélation de la Beauté; trouvera-t-elle cette Beauté dans son amant? ou dans sa propre image? Sera-t-elle capable de l'activité poétique qui réalise la Poésie à travers un être sensible? ou se 
bornera-t-elle à approuver l'oeuvre de son mâle" (8, I, 374375). Ainsi, indépendamment des beaux mythes de la femme, celle-ci "a toujours été, sinon l'esclave de l'homme, du moins sa vassale; les deux sexes ne se sont jamais partagés le monde à égalité" $(8,20)$. En grandes lignes, la soumission de la femme de la part de l'homme s'exprime dans le fait qu'elle, qui est aussi un sujet, une activité, est traitée d'entierement passive, en niant ainsi sa liberté (8, II, 602). Mais ce ne sont pas seulement les hommes et la société qui le font, les femmes aussi ont contribué à cela en acceptant d'être de simples suppléments aux hommes -des proies d'amour, des épouses, des meres, des amantes, des secrétaires etc., au lieu d'assumer les difficultes de leur être. C'est pour cela que Beauvoir souligne qu"on ne naît pas femme: on le devient" (8, II, 13). Puisqu'il n'y a pas une essence ou une "nature" féminine, on doit se constituer comme femme. Autrement dit, ce n'est pas la nature qui détermine la femme, mais c'est la femme qui détermine la nature de fait que "renoncer à sa féminité, c'est renoncer à une part de son humanité" (8, II, 601). Cela implique la nécessité de reconnaître la structure commune des deux sexes et de prendre conscience de la femme non plus comme l'autre mais comme un pour-soi, c'est-à-dire comme activité et liberté.

Avec cet aspect particulier de l'Autre cette oeuvre de Beauvoir pose implicitement les problemes de l'identité et de la légitimation devenus des thèmes centrals pour les postmodernistes. C'est François Lyotard qui a posé ces questions de la façon la plus aigue dans $L a$ condition postmoderne, où il a annoncé la fin des grands récits spéculatifs et universalisants et la dissémination des jeux de langage, des liens sociaux et du Sujet de la légitimation $(16,66-67)$. Dans cette perspective l'autre a cessé d'être un sujet abstrait de la pensée universelle pour prendre dans Le différend la figure de l'autre exclu du système, le plaignant qui étant privé des moyens d'argumentation se transforme par-là en proie. Le différend, c'est cet autre qui se trouve dans une situation dans laquelle les règles pour la résolution du conflit sont établies dans l'idiome de la contre-partie, de façon qu'il ne peut pas prouver qu'il a été injurié $(17,2425)$. Tel, par example, les morts dans les chambres à gaz à Auschwitz ou les proies des procès staliniens qui n'ont pas pu comparaître au tribunal pour dire comment ils ont été tuées. Le fait des phrases et des figures hétérogènes pose aussi bien le problème du sujet de la légitimation que celui de la compréhension. Voilà ce que réplique Lyotard dans ses Moralites postmodernes aux partisans de la praxis communicative tels Habermas et Frank: "Les messages s'échangent à 
condition qu'ils soient compréhensibles... Et à condition que vous et moi puissions occuper tour à tour les positions du locuteur et de l'allocutaire. Richard Rorty va jusqu'à soutenir que cette condition pragmatique suffit, à elle seule, à garantir la solidarité démocratique, sans considération de ce qui se dit ni de la manière de le dire. La langue peut être "blanche", comme celle de L'étranger de Camus, il n'importe que de l'adresser à autrui. Les langues humaines conferent structurellement au locuteur la capacité de parler aux autres. Mais capacité n'est pas devoir. On n'a encore jamais prouvé qu'un silence voulu soit une faute. Ce qui est un crime, c'est de l'imposer à l'autre. On exclut celui-ci de la communauté interlocutrice et, de surcroît, on ajoute à ce dommage un tort plus grave encore, puisque interdit de parole, le banni n'a pas les moyens de faire appel de son bannissement. Politique, social ou culturel, tel est l'exercice de la terreur: priver l'autre du pouvoir de répliquer à cette privation. Quoi qu'on pense, la peine de mort, si légale soit-elle, évoque toujours ce crime. Mais, aussi bien, l'enfant à qui ses camarades disent qu'ils ne joueront pas avec lui et que ça ne se discute pas est en vérité victime d'un crime contre l'humanité" $(18,179)$.

\section{Polyphonie et superposition: \\ Paul Ricoeur's herméneutique de l'Autre}

Comment pourrait-on, sinon réconcilier ces différentes conceptions de l'autre, trouver, au moins, le lien explicatif de leurs raisons d'être? La clé d'une telle entreprise nous est donnée, à mon avis, dans l'approche de la phénoménologie herméneutique de Paul Ricoeur. On pourrait dire, sans exagérer, que ce que le génie de Dostoievsky ạ réussi à faire en litterature, le génie de Ricoeur a réussi en philosophie--rendre la polyphonie des différences, la confrontation des voix, des consciences et des visions differant jusqu'à l'opposé, en dépassant le discours homophonique (Cf. 2, 13-16). Et non seulement cela. Paul Ricoeur a proposé plusieurs conceptions très originales de l'Autre qui dévoilent successivement ses modes différents d'apparition ou d'être--le socius, le prochain, et l'altérité comme élément constitutif de l'ipséité.

Nous avons vu qu'en fait, avant Ricoeur, les philosophes accentuaient soit sur l'autre en tant que alter ego, en tant que "tu" ou prochain, auquel nous sommes liés dans le plus intime de nous-même, soit sur l'autre comme un être différent, duquel je suis isolé ou/et en conflit. Ce n'est que par la dialectique Ricoeurienne du socius et du 
prochain que ces deux images de l'autre en deviennent complémentaires en recevant leur sens véritable et leur explication intégrale.

L'origine de l'idée du prochain se trouve, d'après Ricoeur, dans la parabole évangélique du bon Samaritain. Celui-ci est "une catégorie pour les autres": pour le Juif pieux il est la catégorie de l'Etranger, car il ne fait pas partie de son groupe, c'est un homme sans passé ni tradition authentique, impur de race et de piété,c'est-à-dire un sujet marginal. Et pourtant, par sa conduite il invente la relation directe "d'homme à homme", sans la médiation sociale d'une institution. Sa compassion est un geste au-delà du rôle, du personnage, de la fonction. Elle innove une mutualité hypersociologique de la personne et de son vis-a-vis $(29,100$ 101). Autrement dit, "le prochain, c'est la manière persontelle dont je rencontre autui, par-delà toute médiation sociale" $(29,102)$. On arrive à la contradiclion fondamentale entre socius, le sujet que je découvre de façon médiate à travers sa fonction sociale, et prochain. La question: "Qui est notre prochain" nous met en fait devant le mythe du social. Car la société moderne n'est-elle pas un monde de socius, un monde déshumanisé et de relations abstraites, anonymes et lointaines, un monde sans prochain? Dans une telle attitude anti-moderne l'Evangile annoncerait la condamnation globale du monde du socius comme monstrueuse conjonction de l'usine, de la caserne, de la prison et du camp de concentration. Dès lors le rêve du prochain est contraint, d'après Ricoeur, "de se chercher des signes en marge de l'histoire, de se réfugier dans de petites communautés non techniques et "prophétiques", en attendant que se monde se détruise lui-même..." $(29,103)$.

Mais cette opposition du socius et du prochain se révèle apparente si on les conçoit comme deux dimen-sions de la même histoire, comme les deux faces de la même charité. L'opposition brutale entre communauté et société, entre relation person-nelle et relation administrative ou institutionnelle, ne peut être qu'un moment de la réflexion, car avec la parole: "Rendez à César ce qui est à César, et à Dieu, ce qui est à Dieu" et l'épisode de Jésus disant à Pilate: "Tu n'aurais aucun pouvoir sur moi s'il ne t'avait été donné d'en haut," l'Evangile suggère le prestige spécial de l'institution-l'autorité accordée par Dieu. Aussi decouvre-t-il la charité sous la figure de la justice, ce qui est à voir dans le mot de Paul: "Le magistrat est ministre de Dieu pour ton bien..." (Rom., 13:1-7). Ainsi les figures du prochain et du magistrat ne sont-ils que deux figures partielles ou partiales du gouvernement de l'histoire par la charité. "C'est pourquoi--ecrit Ricoeur-- la croissance du Royaume de 
Dieu se fait dans la douleur des contradictions: le débat, dans notre vie individuelle et dans la vie des groupes, entre les relations "courtes" de personne à personne et les relations "longues" à travers les institutions est un aspect de cette souffrance historique" $(29,106)$. Ce qui importe ici, c'est que Ricoeur accentue pour la premiere fois sur la richesse de la dialectique du socius et du prochain qui peut prendre des formes très différentes: conflit, marginalité, réciprocité. La parabole du bon Samaritain ne signifie pas une opposition absolue entrs le socius et le prochain et ne nous prive aucunement de la responsabilité et de la necessité de penser le prochain dans une situation sociale concrète, resp. de la responsabilité de justifier ou de rejeter une institution. Les institutions constituent en quelque sorte le canevas social des échanges les plus intimes de la vie privée. C'est pourquoi ils peuvent devenir quelquefois un facteur du rapprochement des gens, du fait par exemple que la charité apparaît quand j'aperçois chez l'autre une condition commune de malheur collectif tel que l'exploi-tation coloniale, la discrimination de race, etc. Ainsi le prochain devient-il concret au pluriel et abstrait au singulier. L'opposition entre le socius et le prochain est donc une des possibilites, la plus spectaculaire et la plus dramatique, mais non pas la plus signifiante de la dialectique de la charité. Les institutions sont une menace et un malefice seulement dans la mesure qu'elles contiennent la possibilité d'objectivation des hommes et qu'elles tendent à développer des passions de pouvoir. Dès qu'une oligarchie--technocratique, politique, militaire, écclesiastique--est en place, elle tend à faire de cet outillage un moyen de domination et non de service. Le sens du prochain est donc une invitation à situer exactement les limites du bon et du mauvais usage des instruments, de la technique et de l'appareil administratif. Ainsi, à la differrence d'un Rousseau ou d'un Freud, Ricoeur pense que "le vice de l'existence sociale de l'homme moderne n'est pas d'être contre-nature; ce n'est pas de naturel qu'elle manque, mais de charité" $(29,108)$. La démesure du social vient non des systèmes ou des institutions, mais de l'objectivation de l'homme dans les relations abstraites et anonymes de la vie écono-mique, sociale et politique; le social tend à obturer l'accès et à cacher le mystère des relations interhumaines, et à dissimuler le mouvement de la charité. Cela veut dire qu'il faut chercher le sens final des institutions et des structures sociales dans le service qu'elles doivent apporter aux hommes. Et cela signifie reconnaissance de l'alternative suivante: "C'est l'histoire--et sa dialectique du prochain et du socius--qui maintient l'envergure de la charité; mais c'est finalement la charité qui gouverne la relation du socius et la relation au prochain, leur donnant une commune intention" $(29,111)$. Cette 
intention commune est la tendance vers la Transcendance et se trouve en l'homme même comme imago Dei. Au fait c'est une possibilité offerte par Dieu ",, de nous faire devenir ce qu'il est lui-même", comme disait Irénée $(29,114)$. Autrement dit, de nous faire Amour, de nous faire être-pour-l'autre.

Dans son livre récent Soi-même comme un autre (1990) Ricoeur suggère une lecture tout à fait differente de l'autre, basée sur la dialectique de l'ipséité et de l'altérité. L'idée centrale c'est que l'ipséité du soi-même implique l'alterité à un degré si intime que l'une ne se laisse pas penser sans l'autre. La dialectique de l'ipséité qui révèle celle-ci comme identité-ipse (comme soi) et identité-idem (ce qui reste le même ou "mêmeté") fait apparaître le caractère polysémique de l'altérité, qui ne peut être réduite à l'alterité d'un Autrui: L'autre comme autre que soi, et l'Autre comme pôle du Même. "La vertu principale d'une telle dialectique--dit Ricoeur--est d'interdire au soi d'occuper la place du fondement $(30,368)$. C'est la raison pour laquelle il parle d'un trépied de l'altérité comme expérience de la passivité: L'expérience du corps propre ou chair, la relation de soi à l'etranger comme autre que soi, et le rapport de soi-même qu'est la conscience au sens allemand de Gewissen. En plaçant ainsi la conscience en tiers par rapport à la passivité-altérité du corps, Ricoeur releve pour la premiere fois cette complexité extraordinaire de l'alterité. L'analyse du corps propre découvre l'homme comme agissant et souffrant. L'agir et le souffrir nous renvoient encore à l'altérité. "La plupart de ces souffrances sont infligées--souligne Ricoeur--à l'homme par l'homme. Elles font que la part la plus importante du mal dans le monde résulte de la violence exercée entre les hommes (...) la passivité du souffrir soi-même devient indiscernable de la passivité de l'être-victime de l'autre que soi $(30,371)$. Quant à l'altérité d'autrui elle se révèle sur plusieurs plans. Sur le plan linguistique l'écoute de la parole reçue fait partie intégrante du discours adressé à... Sur le plan de l'action l'autodé-signation de l'agent apparaît inséparable de l'autre qui me désigne à l'accusatif comme l'acteur de mes actions. Et au plan éthique la définition Ricoeurienne même de l'éthique: "Bien vivre avec et pour autrui dans des institutions justes," renvoit au "pour autrui" où l'amitié avec autrui est la condition d'être "ami de soi" et "fait le lit de la justice" $(30,381)$. Elle révèle aussi deux plans dialectiquement complémentaires: le mouvement du Même vers l'Autre, entrevu par Husserl, qui se déploie dans la dimension gnoséologie, et celui de l'Autre vers le Même, esquissé par Lévinas, qui se déploie dans la dimension éthique. "L'assignation à la responsabilité, selon la seconde dimension, 
renvoie au pouvoir d'autodé-signation, transféré, selon la première dimension, à toute troisième personne supposée capable de dire 'je'." Voilà le résultat général de la dialectique croisée du soi-même et de l'autre $(30,393)$.

A ma question d'interview est-ce qu'il y a dans son oeuvre d'aujourd'hui encore place pour l'analyse de l'autre comme prochain, M. Ricoeur m'a répondu qu'il fallait distinguer deux notions de l'autre-celui, qui a un visage et qui peut être un ami, comme expression des relations intersubjectives, et celui, qui n'a pas de visage, auquel nous sommes liés à travers les institutions. Ces deux relations à l'autre sont mises sur le même plan dans Soi-même comme un autre précisément dans la défini- tion de l'éthique. "Je m'oppose--m'a dit Ricoeur--à un personnalisme étroit qui réduirait toutes les relations à l'autre à celle de 'je et tu'. Il y a aussi bien 'tu' que 'chacun'" $(31,17)$.

Ce parcours des differentes visions de l'Autre suffit pour nous permettre de faire les conclusions suivantes. D'abord que dans la philosophie française se forment quelques notions qui sont autant contradictoires que complémentaires et qui esquissent l'Autre comme "enfer", "paradis", "étranger", "face", "socius", "prochain," etc. Ainsi l'Autre est décelé en même temps comme semblable et différent de moi-même, comme extérieur et intérieur à moi, comme obstacle et condition de mes projets. Suivant le role assigné à l'Autre la philosophie française se constitue comme philosophie de la première personne du singulier (la "philosophie du je" de Sartre et de Camus), comme philosophie de la seconde personne du singulier (le "tuisme" de Marcel, de Mounier et de Lévinas), ou comme philosophie de la première personne du pluriel (la "philosophie du nous" de Nedoncelle, Bergson, Chardin) - paradigmes différents qui ne forment un ensemble polyphonique et dialectique que dans la phénoménologie herméneutique de Ricoeur.

On pourrait dire que les aventures de l'Autre montrent qu'elles ne sont au fond que nos propres aventures--nos differentes façons de le rencontrer, de le découvrir et d'entrer en relation avec lui et par-là de nous rencontrer, de nous dévoiler et de nous réaliser nous-mêmes. Et si "comprendre bien quelqu'un, c'est se comprendre bien soi-même" (Shakespeare), cela veut dire aussi que pour comprendre l'Autre, il faut le porter en soi-même. Il faut avoir souffert la maladie et le manque, s'être senti exclu des autres et complètement étranger, délaissé dans la solitude et l'oubli complets. C'est ainsi que pour pénétrer dans la vie 
ouvrière Simone Weil a quitté son poste universitaire afin de devenir elle-même ouvrière et de subir toutes les humiliations et souffrances propres à cette condition--la faim, la fatigue, la peur, la contrainte, l'hostilité, le renvoi. Cela veut dire enfin que jusqu'à ce que l'homme ne commence à voir en l'Autre son soi-même, ce véritable "moi", qui est "le Royaume de Dieu en nous", il vivra en séparation, en conflits et violences, en mal et en malheurs. Reste à savoir, comme l'a bien dit A.-T. Tymieniecka, "si l'on pourrait jamais éliminer ce trait fondamental de l'homme qui est de chercher que son propre bien..." $(37,141)$. Question rhétorique qui témoigne en fait que l'intérêt ultime de toute réflexion sur l'Autre, c'est de nous rappeler que ce n'est pas un Dieu inventé mais nous tous qui sommes responsables des maux, des rancoeurs et des injustices de ce monde.

Si l'Autre existe, c'est parce que nous - toi et moi - l'avons créé.

Lafayette, octobre 1994

YVANKA RAYNOVA

\section{REFERENCES}

1. Abbagnano, N. Philosophie des Menschlichen Konflikts. Hamburg, 1957.

2. Bakhtine, M. La poétique de Dostoievsky. Sofia: 1976 (en bulgare).

3. Beauvoir, S. de. L'invitee. Paris: Gallimard, 1943.

4. Beauvoir, S. de. Pyrrhus et Cinéas. Paris: Gallimard, 1944.

5. Beauvoir, S. de. Pour une morale de l'ambiguite. Paris: Gallimard, 1947.

6. Beauvoir, S. de. Tous les hommes sont mortels. Paris: Gallimard, 1946.

7. Beauvoir, S. de. Les mandarins. Paris: Gallimard, 1964.

8. Beauvoir, S. de. Le deuxième sexe. t. 1-2, Paris: Folio, 1976.

9. Bergson, $H$. Les deux sources de la Morale et de la Religion. Paris: 1932. 
10.Buber, M. Ich und Du. In: Werke, Bd. 1, Kosel Verlag,1962.

11.Camus, A. L'homme révolté. In: Essais. Paris: Gallimard, 1965.

12.Camus, A. Le mythe de Sisyphe. Paris: Gallimard, 1942 (1974).

13.Contat, M., Rybalka, M. Les écrits de Sartre. Paris: Gallimard, 1970.

14.Heidegger, M. Sein und Zeit. Tubingen: Max Niemeyer, 1979.

15.Lévinas, E. Le temps et l'autre." Paris: Quadrige", PUF, (5e ed.), 1994.

16.Lyotard, J.-F. La condition postmoderne. Paris: Minuit, 1979.

17.Lyotard, J.-F. Le Différend. Paris: Minuit, 1983.

18.Lyotard, J.-F. Moralités postmodernes. Paris: Galilee, 1993.

19.Marcel, G. Etre et avoir. t.1-2. Paris: Aubrier, 1968.

20.Marcel, G. Moi et autrui. In: Homo Viator. Aubier, Paris, 1944.

21.Maritain, J. La philosophie morale. Paris: Gallimard, 1960.

22.Merieau-Ponty, M. La phénoménologie de la perception. Paris: Gallimard,1945.

23.Mounier, E. Introductionaux existentialismes. Paris: Gallimard, 1946.

24.Mounier, E. Le personnalisme. Paris: PUF, 1957.

25.Mounier, E. Oeuvres. t. 14, Paris: Seuil, 1950.

26.Nédoncelle, M. La réciprocité des consciences. Paris: Aubier,1942.

27.Nédoncelle, M. Vers une philosophie de l'Amour et de la Personne. Paris: Aubier, 1942.

28.Nédoncelle, M. Le Personnalisme. In: Encyclopédie française. t.19, Paris, 1957. 
29. Raynova, Y. De Husserl à Ricoeur. Ed. de l'Université de Sofia, 1993 (en bulgare).

30. Ricoeur, P. Histoire et vérité. Paris: Seuil, 1955.

31. Ricoeur, P. Soi-même comme un autre. Paris: Seuil, 1990.

32. Ricoeur, P. La refiguration: bilan après quarante ans. Interview avec Y. Raynova (en bulgare). In: Filosofski altenativi, 1993/ 5.

33. Sartre, J.-P. L'être et néant. Paris: Gallimard, 1943.

34. Sartre, J.-P. L'existentiaLisme est un humanisme. Paris: Nagel, 1948.

35. Sartre, J.-P. Huis-Clos. In: Theatre. Paris: Gallimard, 1947.

36. Sartre, J,-P. Explication de L'étranger. In: Situations, I. Paris: Gallimard, 1947.

37. Teilhard de Chardin, P. Le Milieu Divin. In: Oeuvres Pierre de Teilhard de Chardin, t. 4. Paris: Seuil, 1957.

38. Tymieniecka, A.-T. L'expérience créative à la lumière de la phénoménologie de lavie.

39. Interview avec Y. Raynova (en bulgare). In: Filosofski alternativi, 1993/ 6.

40. Weil, S. La Condition ouvrière. Paris: Gallimard, 1951. 